\title{
Assistência de enfermagem frente a mulheres vítimas de violência no Brasil
}

\author{
Nursing assistance to women victims of violence in Brazil \\ Atención de enfermería para las mujeres víctimas de la violencia en el Brasil
}

Recebido: 11/01/2021 | Revisado: 12/01/2021 | Aceito: 17/01/2021 | Publicado: 20/01/2021

Crislene da Silva de Lima
ORCID: https://orcid.org/0000-0003-0551-7298
Centro Universitário Fametro, Brasil
E-mail: crislene.slima@ @mail.com
Samara Deuzuita de Almeida
Centro Universitário Fametro, Brasil
E-mail: samara.dalmeida@ @mail.com
ORCID: https://orcid.org/000-0002-4660-6425
Joissy Carla Correia do Nascimento
ORCID: https://orcid.org/0000-0001-8414-8130
Centro Universitário Fametro, Brasil
E-mail: joissycarla2.jcn@ gmail.com
André Luiz Freitas Nogueira
Centro Universitário Fametro, Brasil
E-mail: andreefreitas@ outlook.com
Elaine da Silva Costa
ORCID: https:/orcid.or/0000-0002-4698-8566
ORCID: https://orcid.org/0000-0003-1059-5514
Centro Universitário Fametro, Brasil
E-mail: elainesilvacostaflor@gmail.com
Regina Oliveira Magalhães
ORCID: https://orcid.org/0000-0002-2516-0110
Centro Universitário Fametro, Brasil
E-mail: reginaenfermeira30@gmail.com
Anderson Lima Cordeiro da Silva
ORCID: https://orcid.org/0000-0001-6777-0622
Universidade de São Paulo, Brasil
E-mail: andersoncordeiro@usp.br

\section{Resumo}

Objetivo: Descrever as condutas da equipe de enfermagem frente a mulheres vítimas de violência no Brasil. Metodologia: Trata-se de um estudo descritivo e exploratotio, de cunho qualitativo de base revisão da literatura, realizada através das bases de dados Literatura Latino-Americana e do Caribe em Ciências da Saúde (LILACS) e Scientific Electronic Library Online (SciELO). Resultados: A análise de 13 artigos evidenciaram que dentre os mais diversos tipos de violência que acometem as mulheres, a violência, física, psicológica, sexual são as mais prevalentes. A enfermagem exerce um protagonismo na assistência as vítimas, tendo em vista, que esse profissional é o primeiro a ter contato a mesma e proporciona acolhimento, ausculta qualificada, bem como o aplica medidas para minimizar os danos causados pela violência. Conclusão: Haja visto os impactos causados pela violência independentemente do seu tipo, a assistência as vítimas requer qualificação dos profissionais para que sejam atendidas as necessidades a paciente, além de minimizar riscos futuros, visando o bem estar físico, psíquico e social da mesma.

Palavras-chave: Violência contra a mulher; Cuidados de enfermagem; Assistência integral à saúde.

\begin{abstract}
Objective: To describe the behaviors of the nursing team in front of women victims of violence in Brazil. Methodology: This is a descriptive study and exploratotio, of a qualitative nature of base literature review, carried through the databases Latin American and Caribbean Literature in Health Sciences (LILACS) and Scientific Electronic Library Online (SciELO). Results: The analysis of 13 articles showed that among the most diverse types of violence affecting women, violence, physical, psychological, sexual are the most prevalent. Nursing exerts a protagonism in the assistance to the victims, having in mind that this professional is the first one to have contact with it and provides reception, qualified auscultation, as well as it applies measures to minimize the damages caused by violence. Conclusion: Having seen the impacts caused by violence independently of its type, the assistance to the victims requires qualification of the professionals so that the needs of the patient are taken care of, besides minimizing future risks, aiming at the physical, psychic and social well being of the same.
\end{abstract}

Keywords: Violence against women; Nursing care; Comprehensive health care. 


\begin{abstract}
Resumen
Objetivo: Describir el comportamiento del equipo de enfermería hacia las mujeres víctimas de la violencia en Brasil. Metodología: Se trata de un estudio descriptivo y exploratorio, con una revisión cualitativa de la literatura, realizado a través de las bases de datos Literatura Latinoamericana y del Caribe en Ciencias de la Salud (LILACS) y Biblioteca Científica Electrónica en Línea (SciELO). Resultados: El análisis de 13 artículos mostró que entre los más diversos tipos de violencia que afectan a las mujeres, la violencia, física, psicológica, sexual son los más prevalentes. La enfermería desempeña un papel fundamental en la asistencia a las víctimas, teniendo en cuenta que este profesional es el primero en tener contacto con ellas y les proporciona recepción, auscultación cualificada, así como la aplicación de medidas para reducir al mínimo los daños causados por la violencia. Conclusión: Teniendo en cuenta los efectos causados por la violencia, independientemente de su tipo, la asistencia a las víctimas requiere la calificación de profesionales para que se satisfagan las necesidades del paciente, además de reducir al mínimo los riesgos futuros, con miras a su bienestar físico, psicológico y social.
\end{abstract}

Palabras clave: Violencia contra la mujer; Atención de enfermería; Atención integral de salud.

\title{
1. Introdução
}

Do ponto de vista histórico e literário, a violência contra mulheres é descrita desde o período pré-histórico perpetuando até a atualidade em uma crescente de novos casos, constituindo um problema de saúde pública e de violação dos direitos humanos, tendo em vista, o seu reflexo nos segmentos físicos, sociais e psíquicos, levando em consideração a compreensão da Organização Mundial da Saúde (OMS) acerca do significado de saúde (Segre; Ferraz, 1997; Carneiro; Fraga, 2012; Souza et al., 2016).

Segundo Moura et al. (2011), toda ação ou conduta que seja fundamentada no gênero e que possa causar morte, dano ou sofrimento psicológico, físico ou sexual, é tida como violação da mulher, sendo em âmbito público ou privado. Nesse sentido, a Lei $\mathrm{n}^{\circ}$ 11.340, de 7 de agosto de 2006, intitulada "Lei Maria da Penha" caracteriza a diversidade de violência doméstica e familiar contra mulher, como: patrimonial, sexual, física, moral e psicológica (Brasil, 2020).

De acordo com os dados apresentados pelo Ministério da Mulher, da Família e dos Direitos Humanos (MMFDH) (2020), o Brasil registrou no ano de 201985.412 denúncias, sendo 78\% relacionada à violência doméstica; 4,35\% ao feminicídio e $61,11 \%$ à violência física. Em 2020, esses casos aumentaram em $27 \%$, sendo reflexo do isolamento social proposto pelas autoridades de saúde pública por conta da pandemia causada pela COVID-19 (Moreira et al., 2020).

Em 2004, o Ministério da Saúde (MS) implementou a Política Nacional de Atenção à Saúde da Mulher (PNASM) que possibilitou a inserção da abordagem de gênero, assim como os cuidados relacionados à vítima de violência sexual e intrafamiliar nos serviços de saúde do Sistema Único de Saúde (SUS), através do Protocolo da Atenção Básica (Moura et al., 2011).

A mulher vítima de violência sexual tem garantia de atendimento coberto pelo SUS, com o objetivo de diminuir os agravos gerados pela violência como: prevenção de Infecções Sexualmente Transmissíveis (IST`s) e gravidez indesejada, de tal forma, os profissionais que atuam frente a esse grupo precisam estar capacitados para que a assistência seja humanizada, a fim de garantir um melhor desfecho quanto ao acolhimento e detecção de possíveis riscos à saúde e integridade da mulher (Lima et al., 2018).

Neste contexto, a equipe de enfermagem exerce atividades de destaque no atendimento as mulheres que passaram por abusos, tendo em vista que esses profissionais são os primeiros a terem contato com as vítimas, acolhendo, orientando, realizando os encaminhamentos necessários e notificando os casos (Silva; Rimeiro, 2020).

Neste sentido, o objetivo da pesquisa foi descrever as condutas da equipe de enfermagem frente a mulheres vítimas de violência no Brasil.

\section{Metodologia}

O presente artigo caracteriza-se por um estudo descritivo e exploratório de cunho qualitativo, caracterizado por 
proporcionar a interpretação por parte do pesquisador com suas opiniões acerca da temática abordada (Pereira, et al, 2018) e de base revisão da literatura, descrita por Souza et al. (2010), como um método que fornece discernimento e apresenta resultados de estudos com uma vasta abordagem metodológica. Com o objetivo de elencar as condutas da equipe de enfermagem no atendimento a mulheres vítimas dos mais variados tipos de violência no Brasil. O estudo foi realizado por intermédio das bases de dados bibliográficas nacionais e internacionais, como: Literatura Latino-Americana e do Caribe em Ciências da Saúde (LILACS) e Scientific Electronic Library Online (SciELO), com auxílio dos Descritores em Ciências da Saúde (DeCS) Violência contra a Mulher; Cuidados de Enfermagem e Assistência Integral à Saúde, bem como dos Descritores Booleanos: AND e/ou OR.

Diante o exposto, foram elegíveis aos estudos artigos completos nos idiomas português e inglês, disponibilizados gratuitamente e publicados nos últimos dez anos (2010-2020). Dessa forma, artigos incompletos, em outros idiomas que não os já aqui citados, publicados anteriormente ao ano de 2010, teses de doutorado, dissertação de mestrado e monografias configuraram inelegíveis ao estudo.

Os artigos encontrados foram organizados em quadros e tabelas, respeitando os critérios de elegibilidade e inelegibilidade da presente pesquisa, considerando título, resumo, leitura na integra e seus resultados alcançados, a fim de seleciona-los para a revisão. A análise dos dados se deu pelo eixo temático intitulado "Violência contra a mulher no contexto Brasil e as ações de enfermagem frente aos casos".

\section{Resultados e Discussão}

O presente artigo aborda uma descrição interpretativa dos achados na literatura, com base nos DeCS, bem como aos critérios de elegibilidade e inelegibilidade proposto para realização do mesmo, após análise somente 13 artigos atenderam aos critérios (Quadro 1), no qual, é possível observar a autoria, título, idiomas, base de dados e o ano da publicação, ressaltando a linha temática da pesquisa "Violência contra a mulher no contexto Brasil e as ações de enfermagem frente aos casos". Proporcionando uma melhor compreensão dos achados acerca dos principais tipos de violência descritos em publicações e as condutas da equipe de enfermagem frente a assistência as vítimas.

Quadro 1. Relação de publicações elegíveis ao estudo após aplicação dos critérios.

\begin{tabular}{|c|l|l|c|c|c|}
\hline \multicolumn{1}{|c|}{ AUTOR } & \multicolumn{1}{|c|}{ TíTULO } & IDIOMA & BASE DE DADOS & ANO \\
\hline $\mathbf{1}$ & ARBOIT et al. & $\begin{array}{l}\text { Atenção à saúde de mulheres em situação de } \\
\text { violência: desarticulação dos profissionais em } \\
\text { rede }\end{array}$ & Inglês & SciELO & 2017 \\
\hline $\mathbf{2}$ & BATISTETTI et al. & $\begin{array}{l}\text { A percepção da vítima de violência sexual } \\
\text { quanto ao acolhimento no hospital de } \\
\text { referência no Paraná }\end{array}$ & Português & Lilacs & 2020 \\
\hline $\mathbf{3}$ & BERNARDINO et al. & $\begin{array}{l}\text { Violência contra mulheres em diferentes } \\
\text { extágios do ciclo de vida no Brasil: um estudo } \\
\text { exploratório }\end{array}$ & Português & SciELO & 2016 \\
\hline $\mathbf{4}$ & BORBUREMA et al. & $\begin{array}{l}\text { Violência contra mulher em contexto de } \\
\text { vulnerabilidade social na atenção primária: }\end{array}$ & Português & Lilacs & 2017 \\
\hline
\end{tabular}




\begin{tabular}{|c|c|c|c|c|c|}
\hline & & registro de violência em prontuários & & & \\
\hline 5 & CAVALCANTI et al. & $\begin{array}{l}\text { A violência contra a mulher no sistema único } \\
\text { de saúde }\end{array}$ & Português & Lilacs & 2020 \\
\hline 6 & CORTES et al. & $\begin{array}{l}\text { Cuidar Mulheres em situação de violência: } \\
\text { empoderamento da enfermagem em busca de } \\
\text { equidade de gênero }\end{array}$ & Português & SciELO & 2015 \\
\hline 7 & CORTES; PADOIN & $\begin{array}{l}\text { Intencionalidade da ação de Cuidar mulheres } \\
\text { em situação de violência: contribuições para a } \\
\text { Enfermagem e Saúde }\end{array}$ & Português & SciELO & 2016 \\
\hline 8 & FONSECA et al. & $\begin{array}{l}\text { Violência doméstica contra a mulher: } \\
\text { realidades e representações sociais }\end{array}$ & Português & SciELO & 2012 \\
\hline 9 & $\begin{array}{l}\text { FORNARI; } \\
\text { LABRONICI }\end{array}$ & $\begin{array}{l}\text { Processo de resiliência em mulheres vítimas } \\
\text { de violência sexual: uma possibilidade de } \\
\text { cuidado }\end{array}$ & Português & Lilacs & 2018 \\
\hline 10 & FREITAS et al. & $\begin{array}{l}\text { Atuação dos enfermeiros na identificação e } \\
\text { notificação dos casos de violência contra a } \\
\text { mulher }\end{array}$ & Português & Lilacs & 2017 \\
\hline 11 & MACHADO et al. & $\begin{array}{l}\text { Violência contra a mulher: o que acontece } \\
\text { quando a Delegacia de Defesa da Mulher está } \\
\text { fechada? }\end{array}$ & Português & SciELO & 2020 \\
\hline 12 & $\begin{array}{l}\text { MASCARENHAS et } \\
\text { al. }\end{array}$ & $\begin{array}{l}\text { Análise das notificações de violência por } \\
\text { parceiro íntimo contra mulheres, Brasil, 2011- } \\
2017\end{array}$ & Português & SciELO & 2020 \\
\hline 13 & OLIVEIRA et al. & $\begin{array}{l}\text { Perfil da vítima e característica da violência } \\
\text { contra a mulher no estado de Rondônia-Brasil }\end{array}$ & Português & SciELO & 2019 \\
\hline
\end{tabular}

Fonte: Lima et al. (2021).

\subsection{Principais tipos de violência envolvendo mulheres}

De acordo com estudo de Bernardino et al. (2016), os tipos de violências mais predominantes e prevalentes, são: físicas, domésticas, seguido de ameaça e agressão verbal. Os autores ainda evidenciam que as práticas agressivas são na sua grande maioria provenientes de seus companheiros, namorados, estanhos, ex-companheiros, ex-namorados e familiares, seguindo essa ordem.

Mascarenhas et al. (2020), reforça com seu estudo que os tipos de violência mais relatados foram os abusos físicos correspondendo a $86,6 \%$; psicológicos com equivalência de $53,1 \%$ e sexuais $(4,8 \%)$, bem como descreve que as notificações de violência por parceiros íntimos são mais prevalentes, além de ocorrerem em ambiente domiciliar. 
Por tanto, as violências de cunho físicas são com maior frequência notificadas e por tanto recorrente. Haja visto, que mulheres são vulneráveis e bem mais frágeis, o que as tornam suscetíveis a tal ação. Oliveira et al. (2019); Borburema et al. (2017), através de sua investigação cientifica corrobora para a afirmação, complementando que as vítimas de violência estão propícias a mais de um tipo de violência e que em sua conjuntura as violências físicas, sexual e psicológicas são frequentes.

Segundo Cavalcante et al. (2020), há uma predominância de notificações acerca da violência sexual, no entanto, descreve que essa prática se dá pela vontade do agressor de reprimir e dominar a vítima, o que contraria o seu desejo sexual, além de elencar, a violência psicológica, moral e violência física como recorrência.

Machado et al. (2020), evidencia que há a existência de um bloqueio por parte das vítimas de violência sexual no momento do registro do Boletim de Ocorrência (BO), os autores atribuem esse comportamento à crença e/ou cultura, por razão de socialmente a mulher ter o dever de satisfazer o seu companheiro sexualmente, o que corrobora para que elas não denunciem.

Em outra perspectiva, Fonseca et al. (2012), elenca a humilhação, xingamentos, desprezos e ataques a auto estimas como fatores relacionados a violência psicologia, descrevendo esse tipo de agressão como um das mais recorrentes. Podendo assim, identificar a opressão machista acerca da mulher. Por outro lado, Cavalcanti et al. (2020), relata que as agressões verbais são dadas como ponta pé inicial para violência física e sexual.

\subsection{Condutas da equipe de enfermagem frente a mulheres vítimas de violência}

De acordo com Cortes e Padoin (2016), a equipe de enfermagem exerce papel fundamental no atendimento às mulheres vítimas de violência, a fim de garantir o cuidado físico e auxiliar na recuperação da saúde da mulher, através de exames, curativos, administração de medicamentos, bem como no planejamento de suas intervenções com intuito de diminuir os danos causados pela violência, como: prevenindo uma gravidez indesejada e as Infecções Sexualmente Transmissíveis (IST's).

Segundo Batistetti et al. (2020), a primeira assistência a mulher vítima de violência, deve ser voltada para o acolhimento e escuta qualificada buscando uma construção de empatia entre profissional e paciente, dessa forma conseguindo transparecer segurança, confiança e credibilidade a vítima, vislumbrando um melhor atendimento e serviço. O profissional de enfermagem deve promover acolhimento, diálogo aberto e flexibilidade. Com intuito de desenvolver e estabelecer uma assistência humanizada e moral (Fornari; Labronici, 2018).

Portanto o acolhimento e escuta qualificada são atribuições do enfermeiro para o melhor atendimento a mulheres vítimas de violência (Cortes et al., 2015). O acolhimento consiste em um processo contínuo e permeado pela escuta qualificada que possibilita entender a situação em que a mulher vítima de violência se encontra, o atendimento deve ser feito desde a chegada da mulher no serviço de saúde até o momento de transferência para outro setor, para tanto, o acolhimento demonstra a proteção e cuidado por parte do enfermeiro (Arboit, et al, 2017).

\section{Conclusão}

Nessa perspectiva, a violência contra mulher caracteriza-se como um problema de saúde pública e pode ser classificada, como: doméstica ou familiar, sexual, física, psicológica e financeira ou patrimonial, entre outras. Sendo as agressões físicas, domesticas, psicológicas e sexuais as mais recorrentes, apresentando uma crescente nos números de casos com o passar dos anos. A enfermagem nesse contexto exerce atividades de acolhimento, ausculta qualificada, rastreamento e prevenção de danos causados pela agressão, bem como busca a reinserção das mulheres na sociedade garantindo o bem estar físico, psíquico e social.

Com o presente estudo espera-se fomentar a produção cientifica, a fim de produzir mais conhecimentos acerca da 
temática, bem como a criação e/ou reformulações de políticas públicas de saúde para garantir a qualidade, aprimoramento e inovações no que tange o atendimento à mulher violentadas.

\section{Referências}

Arboit, J., Padoin, S. M. M., Vieira, L. B., Paula, C. C., Costa, M. C., \& Cortes, L. F. (2017). Atenção à saúde de mulheres em situação de violência: desarticulação dos profissionais em rede. Revista da Escola de Enfermagem da USP, 51, e03207. Epub April 03, 2017.https://dx.doi.org/10.1590/s1980220x2016113303207

Batistetti, L. T., Lima, M. C. D., Souza, S. R. R. K. (2020). A percepção da vítima de violência sexual quanto ao acolhimento em um hospital de referência no Paraná. Rev. Pesqui. (Univ. Fed. Estado Rio J., Online); 12: 168-174.

Bernardino, Í. M., Barbosa, K. G. N., Nóbrega, L. M., Cavalcante, G. M. S., Ferreira, E. F., \& D’Avila, S. (2016). Violência contra mulheres em diferentes estágios do ciclo de vida no Brasil: um estudo exploratório. Revista Brasileira de Epidemiologia, 19(4), 740-752. https://doi.org/10.1590/19805497201600040005

Borburema, T. L. R., Pacheco, A. P., Nunes, A. A., Moré, C. L. O. O., \& Krenkel, S. (2017). Violência contra mulher em contexto de vulnerabilidade social na Atenção Primária: registro de violência em prontuários. Revista Brasileira De Medicina De Família E Comunidade, 12(39), 1-13. https://doi.org/10.5712/rbmfc12(39)1460

Brasil, Ministério da Mulher, da Família e dos Direitos Humanos. Balanço 2019 Ligue 180 Central de Atendimento à Mulher. 2020

Cavalcanti, G. M. B.; Amorim, A. V. B., Queiroz, G. S., Cruz, N. M., Costa, R. L., Bezerra, K. F. O. (2020). A violência contra a mulher no sistema único de saúde. Rev. Pesqui. (Univ. Fed. Estado Rio J., Online); 12: 145-153.

Cortes, L. F., \& Padoin, S. M. M. (2016). Intencionalidade da ação de Cuidar mulheres em situação de violência: contribuições para a Enfermagem e Saúde. Escola Anna Nery, 20(4), e20160083. Epub August 25, 2016.https://dx.doi.org/10.5935/1414-8145.20160083

Cortes, L. F., Padoin, S. M. M., Vieira, L. B., Landerdahl, M. C., \& Arboit, J. (2015). Cuidar mulheres em situação de violência: empoderamento da enfermagem em busca de equidade de gênero. Revista Gaúcha de Enfermagem, 36(spe), 77-84. https://doi.org/10.1590/1983-1447.2015.esp.57162

Fonseca, D. H., Ribeiro, C.G., e Leal, N.S. B. (2012). Violência doméstica contra a mulher: realidades e representações sociais. Psicologia \& Sociedade, 24(2), 307-314. https://dx.doi.org/10.1590/S0102-71822012000200008

Fornari, L., \& Labronici, L. (2018). O PROCESSO DE RESILIÊNCIA EM MULHERES VÍTIMAS DE VIOLENCIA SEXUAL: UMA POSSIBILIDADE DE CUIDADO. Cogitare Enfermagem, 23(1). doi:http://dx.doi.org/10.5380/ce.v23i1.52081.

Freitas, R. J. M. de, Sousa, V. B. de, Costa, T. da S. C. e, Feitosa, R. M. M., Monteiro, A. R. M., \& Moura, N. A. de. (2018). Atuação dos enfermeiros na identificação e notificação dos casos de violência contra a mulher. HU Revista, 43(2), 91 - 97. https://doi.org/10.34019/1982-8047.2017.v43.2585

Lima, F. et al. (2018). Papel da enfermagem na assistência à mulher vítima de estupro. Braz. J. Surg. Clin. Res. V.22,n.1,pp.107-109.

Machado, D. F., Almeida, M. A. S., Dias, A., Bernardes, J.M., \& Castanheira, E. R. L.. (2020). Violência contra a mulher: o que acontece quando a Delegacia de Defesa da Mulher está fechada?. Ciência \& Saúde Coletiva, 25(2), 483-494. Epub February 03, 2020.https://doi.org/10.1590/1413-81232020252.14092018

Mascarenhas, M. D. M., Tomaz, G. R., Meneses, G. M. S., Rodrigues, M. T. P., Pereira, V. O. M., \& Corassa, R. B. (2020). Análise das notificações de violência por parceiro íntimo contra mulheres, Brasil, 2011-2017. Revista Brasileira de Epidemiologia, 23(Supl. 1), e200007.SUPL.1. Epub 03 de julho de 2020.https://doi.org/10.1590/1980-549720200007.supl.1

Moreira, L. E., Alves, J. S., Oliveira, R. G., \& Natividade, C. (2020). MULHERES EM TEMPOS DE PANDEMIA: UM ENSAIO TEÓRICO-POLÍTICO SOBRE A CASA E A GUERRA. Psicologia \& Sociedade, 32, e020014. Epub September 04, 2020.https://doi.org/10.1590/1807-0310/2020v32240246

Oliveira, C. A. B. A., Noronha, L. C., Ribeiro, R. Moreira, K. F. A., Pereira, P. P. S., \& Fernandes, D. E. R. (2019). Perfil da vítima e características da violência contra a mulher no estado de Rondônia - Brasil. Revista Cuidarte, 10(1), e573. Epub 2019.https://dx.doi.org/10.15649/cuidarte.v10i1.573.

Pereira A. S. et al. (2018). Metodologia da pesquisa científica. [e-book]. Santa Maria. Ed. UAB/NTE/UFSM. https://repositorio.ufsm.br/bitstream/handle/1/15824/Lic_Computacao_Metodologia-Pesquisa-Cientifica.pdf?sequence=1.

Souza, M. T., Silva, M. D. \& Carvalho, R. (2010). Revisão integrativa: o que é e como fazer? Einstein (São Paulo). 8(1), $102-106$.

Souza, M, M. S., \& Oliveira, M. V. P. (2016). Violência sexual contra a mulher e o papel do enfermeiro revisão de literatura. UNIT-SE_BIO - Repositório Institucional Tiradentes. 